\title{
Appunti di napoletano antico.
}

\section{I. \\ Il napoletano nell' uso letterario del sec. XV.}

Quando Alfonso il Magnanimo entrò solennemente in Napoli, parve che a capo del suo corteo regale incedesse alto e mirabile lo spirito nuovo della Rinascenza. Spirito che accolto come a sua stanza nella reggia d' Alfonso e del successore, richiamò d' ogni intorno ospiti sapienti, sacerdoti delle antiche muse, filosof, grammatici, poeti, e nel vecchio palazzo angioino memore di Roberto ravvivò la gloria umanistica degli studi. Disceso dalla sua patria umbra, venne Gioviano Pontano sotto la direzione del Panormita a ornare, insieme col vecchio maestro, la nascente accademia ch' egli avrebbe più tardi fregiata del suo nome; e mentre intorno a lui fioriva in un rigoglio di orazioni, di versi, di trattati latini 1' umanesimo, Jacopo Sannazaro gemeva in dolce metro toscano i lamenti di Sincero, e il Chariteo tentava tra il sonar delle corde petrarchesche pur la terzina di Dante. $\mathrm{Ma}$ anche al disotto di questo empireo, l' arte napoletana del quattrocento s' aggirò per più umili cieli, lungo una scala che discende fino al popolo; altri poeti, come il De Jennaro, il Perleoni, il Galeota cantavano in forma non ancor bene disciolta dalla sua invoglia dialettale, con debole imitazione petrarchesca o retorico studio dantesco; il conte di Policastro rimava il doloroso rimpianto dei beni perduti e la serena rassegnazione davanti alla morte; altri ancora nella ballata faceva eco alla contemporanea poesia spagnuola penetrata fra noi, - nello strambotto fissava $i$ canti del volgo. Diomede Carafa e Giuniano Maio scrivevano trattati in volgare, in volgare favoleggiava Francesco del Tuppo editore di Dante, e una numerosa famiglia di cronisti narrava nel suo comune dialetto aulico le vicende del regno. $\dot{E}$ tutto un mondo letterario, su cui le ricerche degli ultimi tempi hanno gettato molta luce; un mondo rivelantesi nelle forme più disparate, e perciò degnissimo dell' indagine erudita sotto il rispetto della storia letteraria non meno che della storia linguistica.

$\mathrm{Ma}$ io non so dire se lo studio di quel volgare potrebbe dirsi un capitolo di storia del dialetto napoletano, o non piuttosto della lingua italiana. In tutte le parti d' Italia che non fossero 
Toscana, quella relativa unità che si poteva allora raggiungere non s' ebbe da principio se non per que' generi letterari che riproducevano modelli toscani: il sonetto, la canzone, la novella; forme in cui l' imitazione letteraria genera l' imitazione linguistica, mentre in altri generi più indipendenti l' influsso del toscano o la verniciatura latineggiante giungevano appena a mascherar le violenze dialettali. Questa condizione di cose, comune a tutti i paesi d'Italia, si ha pur nella letteratura napoletana del '400; ma già la troviamo iniziata nel secolo precedente.

Quando Bartolomeo Capasso, maestro dottissimo delle nostre storie, volle mostrare pur con qualche argomento glottologico la falsità dei Diurnali di Matteo Spinello da Giovenazzo, accennò in poche parole le sorti letterarie del dialetto, fermandosi a noverare da un lato i documenti più popolari, dall' altro la molteplice serie delle scritture più colte. E dopo avere accennato alla lettera napoletana del Boccaccio e alle scritture trecentesche pubblicate del Mussafia, dal Miola, dal Percopo, egli passa - a traverso la Cronaca di Partenope - al secolo successivo; nel quale oltre le opere più propriamente letterarie fiorivano nella libera audacia del dialetto $i$ Gliommeri di Jacopo Sannazaro e le farse del Caracciolo, accanto alle scritture volgarmente auliche tra cui si comprendono „le cronache ed memoriali storici, talune traduzioni dal latino, alcune leggi, bandi, statuti ..., le deposizioni dei testimoni e qualche altra composizione somigliante sia in prosa che in versi. Scritture tutte non dettate propriamente nel puro dialetto, $\mathrm{ma}$ in un linguaggio il cui fondo è un italiano, o un dialetto letterato comune a tutte le regioni del regno ...., nel quale si trovano frammiste, dove più, dove meno, voci, o inflessioni di voci, e certe forme grammaticali proprie ai dialetti della provincia cui lo scrittore apparteneva."i

Ma su questa materia sarebbe opportuno intendersi un po' più precisamente. Dicendo che il fondo delle nostre scritture è un dialetto letterato comune a tutte le province del regno, si vien quasi a intendere che realmente, consapevolmente corresse pel mezzogiorno continentale una lingua comune, come credeva il Galiani; per il quale "pugliese" significava non solo il parlar della Puglia, ma l' insieme dei dialetti meridionali nell' uso letterario. "Semplicità grande - scrive il Galiani - sarebbe il credere che questo linguaggio usato negli atti pubblici della Nazione e del $\mathbf{R e}$ non lo fosse stato altro che per ignoranza della buona lingua italiana ... Credasi adunque che il dialetto napoletano fu consecrato da' nostri $\operatorname{Re}$ Aragonesi agli atti pubblici ed alla legislazione, unicamente per decoro della nazione e perchè non si credeva allora che dovesse anteporsegli il Toscano, non gid perchè mancasse chi potesse scrivere nel dialetto toscano." 2

1 Ancora i Diurnali di M. S. da G., Firenze, 1896, p. 18.

2 Del dialetto napoletano, p. Ior. 
Ma se queste opinioni dello spiritoso abate importano soltanto alla nostra curiosita storica, sarebbe invece da ascoltare con la debita riverenza il. Torraca, quando scrive: „... il volgare nella corte di Napoli ... meriterebbe lungo studio." Il movimento fu iniziato dai sovrani e dai principi aragonesi ... Vagheggiavano un ideale di lingua letteraria e speravano formarla purificando il dialetto e fondendo in esso elementi latini." I

Si potrà convenire in tal giudizio? L' uso aulico del dialetto fu consacrato, assai prima che dai re Aragonesi, dal libero impulso del sec. XIV. Lo scrivere a quel modo era un fatto impulsivo, regolato dalla vasta e cosciente efficacia livellatrice del latino, da quella più indeterminata e meno cosciente del toscano. Insomma, avveniva a Napoli quel medesimo che in tutto il resto d'Italia. E naturale poi che da quella confusione sorgesse non già una lingua, ma qualcosa che avendo a suo fondamento il dialetto non era più dialetto, senza che lo scrittore però credesse di fare opera nazionale ribellandosi alla toscanità. Al più, quell' uso letterario cosi incerto potè acquistare per qualche tempo un valore ed un' autorità che diremo tradizionali, e che particolarmente riuscivano a mantenersi in luoghi che fossero, come Napoli, un centro politico.

Ma se ci volgiamo a considerare quegli scrittori che di proposito miravano alle forme superiori dell' arte guardandole nei modelli di Toscana, la vecchia illusione provinciale si sfronda del tutto, nel trionfo incontrastato della buona lingua. Chè se d'ogni intorno sbocciavano negli orticelli poetici $i$ fiorellini dischiusi al sole del Cansoniere, questo fu il più potente impulso letterario a quell' unità linguistica cui pure concorrevano tante e tante altre ragioni storiche o accidentali. Cosi fu che fin dal' 300 si ebbe una scuola toscaneggiante di petrarchisti; così fu che il Sannazaro, piena la mente d' ispirazioni petrarchesche, sorretto anche dal gusto finissimo, potè comporre uno de' più armoniosi monumenti di nostra lingua. Chi confronti le successive edizioni dell' Arcadia può vedere in atto, fra le varie correzioni, quell' affannoso adattarsi alla favella riconosciuta ne' suoi diritti di lingua, ed osservar la cura diligente posta nel ridurre il colorito dialettale. Lo stesso va detto per il Chariteo, il qualo ando cancellando dalle sue rime tutte le parole che - o fossero spagnuole o napoletane o latineggianti - non rispondevano al tipo ch' egli vagheggiava e ch' era appunto il toscano del Petrarca (perchè assai minore e secondaria fu in lui l' influenza di Dante). Una simile tendenza mostrano $i$ canzonieri di Jacopo de Jennaro, del Caracciolo, del Perleoni; ai quali tuttavia non bastaron le forze per levarsi con ali sicure, ma che pur si allontanano di molto, in diversa misura, dal comune linguaggio del tempo. Invece al dialetto s' accosta più quella grossolana e mediocre raccolta poetica dei Rimatori napoletani del 400 messi in

- Studi di storia letteraria napoletana ... 
luce dal Mandalari di su un codice parigino, 1 che di volta in volta $s^{\prime}$ ispirano or dal popolo or dalla poesia spagnuola, 2 senza avere innanzi un modello letterario toscano.

Alquanto minore, e limitato alla prosa, fu l' influsso del Boccaccio, ma efficace anche questo. Se nelle sue novelle ritrasse Masuccio Salernitano direttamente dal Boccaccio i modi della lingua e dello stile, Jacopo Sannazaro contemperò nella sua maggiore opera l' imitazione linguistica del Petrarca e del Boccaccio, ma con manifesta prevalenza di quest' ultimo. Sono inoltre degne di molto studio come minori esempi d' imitazione boccaccevole nello stile, e spesso nel contenuto, quelle numerose lettere che si rinvengono in vari codici del tempo ${ }^{3}$ : lettere d' amore per massima parte, come son quelle del codice parigino contenente rime di napolitani, a cui s' aggiunge una corrispondenza fra il De Jennaro e il conte di Popoli, che fu a quel tempo Giovanni Cantelmo. Il primo manda de' versi, il secondo loda e ringrazia e si lagna per la lunga assenza dell' altro. Ma se anche in queste ultime lettere è sempre evidente il proposito di conformarsi alla sonora ricchezza del Boccaccio, quelle ove si tratta d'amore ricordano specialmente alcune epistole di lui. Una ve n' ha, per esempio, in cui si discute sottilmente di casistica amorosa, come in quelle pagine squisite che vanno innanzi al Filostrato:

„Amantissimo amico, essendo io stato da una donna domandato d' alcuni dubij $e$ in verità secundo el mio basso intellecto multo difficili, avante che presuma risponderli delibero el vostro judicio sentire... El demando dunque de questa donna se fo: primo, se una essendo iovene e nobili de natura, poco men che non maritata havendo da stare al mondo, doveva inamorata vivere o non. El secondo, se haveva da vivere inamorata, in che modo se deveva inamorare .... ecc."

Certo osservò bene il Flamini 4 che a questa fioritura epistolare non fu estranea la fama di Ovidio; ma più direttamente l'autorità del Boccaccio stava sempre innanzi agli occhi degli scrittori di queste esercitazioni letterarie. "Recepute le lictere de vostra Signoria et huudito el suono de quelle non meno da importare la dolczeza et a me la nova eloquencia che odendo el limato dire del fiorentin Boccaccio ..."; parole di colore oscuro, e guaste, ma tali da mostrare che il conte di Popoli è lodato per una lettera in cui pareva d'ascoltare "el limato dire" dell' antico maestro. Altra volta è il conte di Popoli a scrivere che alcuni versi dell'altro „se possono dire procedere da lo famoso Boccaczo fioren-

1 Caserta, 1885.

Savj-Lopez, Lirica spagnuola in Italia, in Giornale storico della letter. ital., vol. XII.

3 Di alcune da notizia il Mazzatinti, Manoscritti italiani delle biblioteche di Francia, I, 104; II, 124-129; di altre - del Galeota - F. Flamini im Giorm. stor. d. Lett. it., XX, p. 46. Buon numero ve $n^{\prime}$ ha nel codice Riccardiano 2752.

- L. cit. pag. 46. 
tino, quale dicite avere letto." Si era parlato, per queste lettere, di derivaxione pagnuola:1 ma io credo piuttosto che sia per la Spagna derivarione italiana. La provenienza italiana di qualche codice catalano e spagnuolo che contiene scritture consimili ci mostra chiaramente da qual parte venisse l'impulso; d' altronde l' imitazione boccaccesca è cosi palese, dirò anzi cosi artificiosamente palese, che non è possibile disconoscerla.

Un po' diversamente stanno le cose per l'Esopo di Francesco del Tuppo, vasta composirione d' indole più morale che artistica, la quale, se pur qualche novella sia derivata dal Decamerone ${ }^{2}$, proviene in generale da ben diverse fonti; e l' autore medesimo avverte in principio dell' opera: „Lectore, per la mercé di Dio è venuta la fine del trabaglio mio, quale, como megliore ho saputo, con basso et umile stile me so portato." Si potrebbe credere che dal suo ,basso et umile stile" il nostro favolatore napoletano non sapesse davvero, anche volendo, salire: se leggiamo la lettera ch' egli premise alla sua edizione della Divina Commedia. Lettera ch' è uno dei più caratteristici documenti dialettali, proprio là dove più spontaneo sarebbe dovuto venire l' impulso a un rinnovellato uso linguistico dal grande modello cui veniva il Del Tuppo consacrando le sue cure editoriali.

E singolare che l' imitazione dantesca, la quale ispirò oltre un poema del De Jennaro anche il Giardeno di Mariro Jonata agnonese, non sapesse a costui ispirare l' imitazione della lingua; chè anzi il Giardeno è ingenuamente sovraccarico di idiotismi provinciali. Fu proprio, ripeto, il Petrarca quegli che con il Boccaccio maggiormente influì sulla diffusione della lingua, sull'adattarsi delle parlate locali al toscano, e più il primo che il secondo. Come si spiega questo? Da una parte, io credo, con la relativa povertà del linguaggio poetico del Canzoniere - povertà è in questo caso facilita - cui 's' era gia fino a un certo punto abituati dalla lirica cortigiana delle varie province; dall' altra col fatto che l' imitasione del Petrarca fu spesso così servile, da potersi dire piuttosto una parafrasi, e quanto più servile è l'imitazione tanto maggiore viene a essere l' adattamento linguistico. Invece l' imitarione di Dante e del Boccaccio era meno intrinseca, si svolse con più liberi modi, lascio allo scrittore campo di spaziare con le sue idee, se ne aveva, e con la sua lingua fuori del solco segnato.

Ma certo è che nel "volgare aragonese" di Napoli si veniva sempre più diffondendo la lingua italiana, senza trovare altri ostacoli che nell' ignoranza o inesperienza di coloro che s' accin-

1 B. Croce, Ricerche ispano-italiane, I. Napoli 1898, pag. 26.

2 Una fonte boccaccesca $\mathrm{e}$ indicata dall' autore - fab. 4I, confirmatio exemplaris: "Secundo scrive messer Johanne Boccazio poeta singularissimo, ill' era una donna che factose venire in casa uno suo amante perchè el marito era absente ... ecc." 
gevano a maneggiarla, e in una grossolana consuetudine delle forme dialettali, che non fu mai deliberata e cosciente opposizione al toscano. Cercando, si trovano a ogni passo imitazioni, citazioni, reminiscenze; perfino quel rozzo scriba disadorno che è Loise de Rosa discute se sia vero "chello che dice Dante, che dice nonn-ey maiure delore che recordare delo tienpo felice indela miseria." Sotto l' usbergo della poesia, il toscano s' insinua minaccioso fra' ripari del dialetto, ne mina con paziente opera l' organismo già scrostato dalle influenze latine, getta nuove schiere di parole nel vecchio castello sguarnito, e lotta e insidia e distrugge, fino all' ultima vittoria.

$\mathrm{Da}$ quanto son venuto finora esponendo, si vede qual compito deva imporsi colui che giovando insieme alla storia del dialetto e della lingua consacri uno studio glottologicamente minuto alla letteratura napoletana del secolo decimoquinto. Egli avrà innanzi a sè una serie vastissima di documenti diversi fra loro per infinite gradazioni e sfumature di lingua; serie che va dalle più rozze scombiccherature volgari alle più squisite opere letterarie, così che queste si trovino come al sommo d' una scala, a cui quelle s' aggrappano invece faticosamente nel vivo sforzo dell' ascesa. Fra tanta abbondanza basterebbe scegliere non più di due o tre diverse scritture, nelle quali fossero variamente rappresentati i caratteri essenziali dell' uso contemporaneo; ed avendo prima studiata quelle più vicine al dialetto, considerare nelle altre più elevate l' azione parificatrice del latino e del toscano.

Alcuni anni or seno G. de Blasiis pubblicò nell' Archivio storico naboletano alcuni frammenti tolti a un codice della Biblioteca Nazionale di Parigi, facendoli precedere da una breve notizia dell' autore.1 Sebbene risalga a un' età piuttosto recente - fu scritta a riprese dal $145^{2}$ in poi $^{2}$ - quest' opera d' uno spirito bizzarro è degna di altissima considerazione, non già per il contenuto, che quale documento storico è insignificante, ma per la lingua: perchè senz' alcun dubbio è questo il più notevole documento dell' antico dialetto napoletano.

Il povero Loise, sebbene citi Dante e pretenda d' aver servito in altissime cariche non so quanti re e regine, è poi un ingenuo ignorante che maltrattato dalle donne e dalla fortuna si decide, vecchio, a raccontar tutte le grandi cose che ha vedute nella sua vita avventurosa: fatti politici, casi personali, faccende di signori, miracoli, aneddoti, storielle d'ogni maniera; e tutto questo alla rinfusa, rozzamente, ma con un tòno di gravità e di ammaestra-

1 Anno IV, fasc. III, pag. 4 II $\mathrm{sgg}$. Pozzuoli.

- Ma il de Rosa era nato, come afferma egli stesso, nel 1385 a 
mento, come un vecchio nonno che 8 ' intrattenga a veglia coi nipotini. Fa ad ogni paseo citaxioni latine come questa: „Et murio,

- et omnis murimur. - Beatissime pater, audiatis dua verba. - Tu cuoche facche (quoque fac) simelem ... ecc.; cosi via. Discorre con qualche interlocutore immaginario o addirittura fa parlare in prima persona i personaggi della sua storia; come quando dà un esempio della generosità d' Alfonso col dire che „una volta le fo cercata la regina, et non sappe dire de no, se no che disse che cercava troppo, disse in catalano mas chiere." Ora, s' intende come in un simile scrittore il dialetto potesse, meglio che altrove, mantenersi immune da troppe deviazioni semidotte e letterarie; e come lo studio di lui sia, per questo rispetto, d' una grande importanza. Le pagine che seguono contengono appunto la descrizione del suo linguaggio - prima pietra del lavoro più vasto che da me o da altri potrà farsi. Da quel moltissimo della Cronaca che ancora oggi è inedito, ho scelto poi alcuni frammenti che bastino a rendere un' immagine di quel povero cortigiano novantenne - il quale, raccogliendo nelle sue pagine tante ingenue voci del secolo, non sapeva di preparare alla scienza ventura tante preziose voci del dialetto.1

\section{II.}

\section{La lingua di Loise de Rosa.}

Vocali toniche.

1. A. nata 'nuota'; i soliti chiove 'chiodi' e alliegro, allegra. Gallicismi: contea, libberea 'livrea'. Con scambio di suffisso insaciebbele.

2. -ARIO, -ARIA. Piu frequente la risoluzione indigena -aro, -ara che non l' importata -iera, -era. Es. I. coiraro, ferraro, rammaro, pintraro; affathuchiara, migliara. Pl. denare. II. osstiere, pannettiere, tresauriere ecc. Fem. pl. camarere. ${ }^{2}$

1 Finchè la Societa Storica Napoletana non abbia curata un' edizione completa di Loise de Rosa, in modo che tutti possano aver davanti il testo, gli appunti che seguono basteranno a darne un saggio preciso e abbastanza copioso ai dialettologi. Essi contengono uno spoglio dell' intero codice, di sulla copia che ne fece eseguire anni sono la Societá Storica; copia che io ho ragione di ritenere assai fedele. Quando feci per me questo lavoro, non curai di notare la collocazione delle singole voci nelle pagine del manoscritto, ed esitavo perció a darlo in luce. Ma poi, considerato che per ora la Società Storica non si accinge alla stampa, nè dà segno di proporsela in avvenire; considerato inoltre che nessuno studioso migliore di me ha posto finora gli occhi sul testo, non ho voluto lasciar piu lungamente dormire questo materiale cosi importante e curioso. Sono modesti appunti: ma sia detto a loro scusa che non pretendono essere di più.

2 Piena conformita dunque con l' uso moderno. Invece altri scrittori del tempo hanno -ero da -ariu, per influenza spagnuola. Del Tuppo cancellero; 
3. $\overline{\mathbf{E}}$ Metafonesi regolare: viro, priso, sivo, pino plēnu, piso, commico, consico; Lorito, Faito. Rarissime le eccezioni come vero ecc. Pl. ry, tappite, pine plēni. Invece legie lēges che in Del Tuppo, Cron. di Part. ecc. è ligi o liggi. Seconda persona: dive dē bes, cong. digie ecc. ${ }^{1}$ Vinde trascina seco attinde, intinde da tĕnd-. Al reg. fice fē ci risponde quasi sempre nella $3^{\mathrm{a}}$ fece, e solo raramente fice. Per anal. della $I^{2}$ pers. sing. ficimo, ficemo - ma fecero; cfr. cresero 'credettero', ecc.

$\mathrm{Fa}$ eccezione all' uso comune sisero 'scesero'. Da -ENSIS regolarmente paese, francese, pl. francise, angrise 'inglesi'; ma una volta sing. marchise accanto al fem. marchesa.

Crodele suonò, come doveva, con $e$, se lo avviciniamo al pl. fidile. Il pl. fem. piacivele, rencressivele sarà per scambio di suffisso.

Pel part. lēctus anal. liesseto, lesseta; elesseto risente del semidotto eletto cui sembra doversi l' assenza del dittongo:2 - Erta nella frase all' erta aveva regolarmente $e$ perchè troviamo insieme all' irto, contro la pronunzia aperta del toscano.

$\mathrm{Da}$ notare frieno frēnu. Trovo un frado, apparentemente nel senso di fretum "como fui alo frado de Isschia." Sarà da leggere fredo $^{3}$ pensando a una forma semidotta? Fretum non è ignoto ai dialetti italiani.4

Innanzi a vocale: creo.

4. $\breve{E}, \mathrm{AE}$. Dittongamento normale; citerò soltanto assiequio *exsequium per exequiae, lieso laesu, siege 'seggi' accanto a segie 'sedie'. Così in posiz. lat. o romanza tienpo templu, siessto, patierno (ma eterno), prisiento 'dono', mienbro (fem. la menbra); essienpro e essenplo, pl. fierre, sing. ferro, prociesso e processo, apriesso e appresso. Plurali pieccze 'pezzi', priece pretii, diffiette. Propaross. miedico, pl. prievete. Raro il mantenimento; vecchio, pl. viecchi; bello, mentre la lettera napoletana del Boccaccio ha biello, biegli.

Regolarmente dittongano -ellu, pl. -elli; -mentum, pl. *-menti. Da - eriu messtiede e minessterio (mistero in Del Tuppo; cfr. Caix, Orig., 71). In leparo lepore il passaggio di decl. è avvenuto troppo tardi perchè potesse aversi il dittongamento.

Nei verbi non viene mai meno il dittongamento regolare della seconda persona: contiente, liege pres. e imper., vieste, inpromiette ecc. $\mathrm{I}^{2}$ pers. credietti. Dittongo anomalo, per influsso di altre forme, in essiegua, e solo grafico nel pl. fem. cierte.

Arro 'erro' è dovuto alle forme arizotoniche, in cui facilmente la liquida richiama $a$-innanzi a sè.

Di Majo cavalero; Codice Aragonese cavalero abalestero cancellero thesaurero e thesaurere. Pl. -eri : cancelleri.

1 Faciva $3^{e}$ pers. errore di scrittura, lo stesso direi di catine pl. di catena; se non vogliamo unirlo a tila tela che si legge nel glossario dei Monumenta Neapolitani ducatus editi da B. Capasso.

In popolare liesseto conferma che letto già nel Regimen sia un latinismo.

8 Cosi si legge nell' Arch. stor. nap., vol. cit.

4 Frieto in una cronaca veneziana: Arch. III, 279.

Zaitschr. $L$ rom. Phil. XXX. 
Tra le voci dove e è normalmente conservata, noto solamente bene: perchè i molti codici fiorentini che conservano la lettera del Boccaccio hanno invece bien che non è altrimenti documentato nel napoletano, in cui sarebbe inesplicabile. $O$ fu un gallicismo nato nella corte angioina, o un errore per falsa analogia, come puorta nella medesima lettera. 1

Come spiegare il costante deice decem? Perchè il nap. mod. non ne ha traccia, bensi il leccese (Arch. IV, Vocalismo leccese, n. 16).

Indeclinabili meglio, pejo.2

Innanzi a vocale: co e yo, dio, mio pl. miey, mey; iebbeleo 'giub.', iodea; pl. iodey e iudiey; la $2^{2}$ pers. di 'essere' è talora siey, ma piu spesso sy.

5. I. Metafonesi regolarmente osservata: pilo, frito con errore grafico per 'freddo', stritto, sinno signu, dinno, cinno 'cenno', vinte viginti, vippeto bibit $\mathrm{u}$, inf. vevere. -Iscu: todissco e tedessca, grecissco. -Issimu conserva la vocale originaria: singularissemo. Fameglio è formato su fameglia. Per cariccze, furticcze v. il mio studio sul suffisso -ities nell' ant. nap. in Ztschr. f. rom. Phil. XXIV, 504. Littere pl. pare voce latineggiante; ma se l' accostiamo al singolare lettere, vediamo che le cose stanno diversamente. Sarà un litterae fatto al sing. la lettere, col pl. in -i?

Regolare è il passaggio a -e- quando non è consentita metafonesi: seta sitis, semele (che d̀ anche del Regimen), marelema ma ritima; fammeglia, commencza, conseglia, lengua, meucza 'milza'.

-Issima, -issimae bonessema, illustressema; veresseme. - -Ibile possevele; ma pl. fem. rencressivele.

1 Qualche appunto a proposito di gallicismi. De retro è qui come negli altri testi napoletani, dereto; cosi endreto del conte di Policastro, arreto nei Bagni: dreto nella Cron. di Partenope a stampa. Bene a ragione dunque il Mussafia ritenne importati arrieri e arrieri, e saranno gallicismi; per cui va ricordata l' opinione del Flechia (Arch. II, 33) che l' elemento francese abbondi nel napoletano più che altri non creda. Certano e cambra trovo in un poemetto napoletano dell' ultimo quattrocento (Isabella del Balso regina di Napoli in un inedito poema sincrono, per cura di B. Croce, Napoli, 1897) mentre il De Rosa ha sempre camara, camera. Di gallicismi è ricca la Cronaca di Partenope dove trovo, per esempio, forgiare e gagia 'gabbia'. Nella Cronaca stessa sono da ricordare per curiosità un pajo di luoghi francesi; che cito dal ms., perchè nella stampa appariscono sformati. Carlo d' Angio, prima di muovere in battaglia contro Manfredi, dice: "Venus est li hore que nus avons tant desiret." E quando gli propongono di seppellire il nemico ucciso, risponde: "Lo feist je volonters, se il ne fuist scomune."

2 Questo è sempre un problema. Il Subak (Die Conjug. im Neapol., Wien, 1897, p. 6) pensò che ,wohl - u (m), aber - u s nicht umlautet, natürlich mit Ausnabme der Neutra der corpus-Classe, die ja zur murus-Deklination gehören." Si vegga anche Meyer-Lübke, Gr. I, 643, e Ascoli, Arch. XIII, 302. L' antico uso letterario fa talvolta eccezione per mino che si trova, per esempio, nel Codice Aragonese - ma è un latinismo; contro l'opinione del Meyer-Lüble, che vorrebbe l'Umlaut soltanto da -us, si potrebbe ancora citare viro del De Rosa nel costrutto verum est. 
Degli indeclinabili noto meno.

I pronomi maschili: isso, chisto, chillo, fem. chesta, chella stanno accanto ai neutri con -e-: chesto, chello. Molteplici teorie hanno tentato di spiegare quest' anomalia. Il Mussafia, nelle sue note al Regimen, n. 13, disse che non avendosi ne' neutri un plur. con metafonesi per $-i$, è mancata al singolare la spinta analogica da cui si ebbe la metafonesi in quei singolari con $-u$ cui stava da canto un pl. con $-i$. Ma dove si attinge la sicurezza che $-u$ non produca metafonesi spontanea, senza l' analogia della corrispondente forma con -i? Il Meyer-Lübke trovo qui invece una conferma alla sua ipotesi, che solamente - us e non -um generi l' 'Umlaut'; mentre l' Ascoli vide in codesti neutri una composizione con hoc. Meno incerta fra tutte mi sembra l'opinione del d' Ovidio, che chello, chesto siano antichi femminili con l' ellissi del nome cosa, o antichi plurali neutri; in entrambi i casi $-a$ si sarebbe affievolita. Ma quale esempio ha il napoletano d' un affievolirsi, di $-a$ che si riduca a -0 ? Io penso che ammesso un remoto neutro chesta, applicato anche al singolare - tanti avanzi neutrali ha il napoletano! - se n' è ricavato chesto per analogia dei singolari in -0 , rimanendo immutata la vocale tonica del neutro originario. Qualcosa di simile, almeno in apparenza, è accaduto nel termine spregiativo del volgo napoletano: fesso. Perchè rimane é? Evidentemente si diceva dapprima: tu si na fessa, una donna, una sciocca; ma riferendo ad uomo quella voce, se ne fece un maschile, lasciando la tonica dell' antico femminile.

Midesimo, infine, è voce letteraria (medemo nei Bagni, mediemmo nel cod. laur. XXVI, pl. XLIII della lettera napoletana del Boccaccio. - Paricchie ha costantemente -i-, anche accompagnato dai neutri femminili centenara, migliara: la metafonesi è dunque dovuta al nesso palatale, indipendentemente dalla finale.

Qualcosa da notare nelle forme verbali: se cirche 'cerchi' è regolare, non tale può dirsi circaro; cfr. Arch. IV, p. 148. Accanto a miette regolarmente l' imper. mittelo. Letteraria sarà la $3^{a}$ pers. intendisse contro il solito -esse.

Innanzi a vocale: veo; corree; tu sie, sia.

6. $\overline{0}$. Anche qui regolarmente osservata la metafonesi. Noto Ottrufo *Octobru, ${ }^{1}$ sulo, vuto; groriuso, ammeruso; ${ }^{2}$ curreturo 'corridojo'; pl. rellegiune, serveture, inperature ecc.

A parte duono, pl. duone, che tirano seco duond accanto a dond. ${ }^{3}$ Invece è normale dute ${ }^{*}$ doti per dotes. Muote è foggiato sulle forme dittongate di mǒvere: muove, muosso; la forma regolare è in terramuto, teramuto, perchè in combinazione si è sottratta - al

1 Octubro e il semid. Octobro ha il Regimen. Tuppo.

2 In nessun altro testo napoletano è cosi costante -uso: si vegga in Del

Del Tuppo, italianamente, doni. Deve trattarsi anche nel De Rosa di voce letteraria, malamente adattata al dialetto. 
contrario della corrispondente voce italiana - all' analogia verbale. In nomo il passaggio di declinazione avvenne troppo tardi perchè ne seguisse l' assimilazione vocalica. Plurali semidotti: nobbele, sole (sing. sulo). II $\mathrm{II}^{2}$ pers. conussie, $\mathrm{I}^{2}$ pers. conossco.

Nella frase nullo piglierrite presune l' anomalia è solo apparente: presune $\mathrm{e}$ in realtà un plurale del solito presone. ${ }^{1}$

Fuori delle condizioni per l'umlaut, sempre - $o$ - fuor che in maiure sing.: maiure delore, to frate maiure. ${ }^{2}$

7. Ǔ. Sempre ben distinto l' esito a seconda della finale: pucczo, subburcu sepulcru, burgo ecc.; pl. iuvene, piducchie, burpe 'volpi'; iovene sing., iodece, doce dulce ecc. chiuputa.

A parte li yorne. Da plov-chioppeto, accanto al part. debole

Assai rare le voci semidotte come secunda, redutta, fusse. Innanzi a vocale doe, dove duae, duy; tuo, toa, toe, suo ecc.

8. o. Costante e rigorosa, come sempre, l' azione della finale. Muodo, luoco (ma regolarmente loco illo-loco), muolo, suodo solidu, puopolo, cuoiro, fuorcze forsit, bruodo; pl. suone, cuoce. Semidotti ioco, pl. omene. Cosi in posizione: cuollo, accuoncio, duosso, gruosso, cuorpo e corpo corpus, cuorpo colaphu pl. cuorpe e corpe, muorcze, apuostole, quattuordice, puorce. $2^{2}$ pers. pruove, duorme, truove, puoy; ma vole *volis accanto a voy, vuy. Semidotti nostro, vostro, pl. vostre accanto alle forme regolarmente dittongate. Sing. e pl. occhie.

Duonno si trova insieme con donno; quest' ultimo proclitico. Il pl. cuonte *comiti testimonia per il sing. conte; invece munti 'monti'.

Accanto a puosto le forme semidotte poseto, epposito. Si alternano respuosse e resposse; segno che non è avvenuta la deviazione analogica di $g$ in $o$ come in rispose. Inturno accanto al regolare contuorno e forse dovuto a proclisia.

-бlus: Piccsulo Puteoli (cfr. i pl. citrule, fasule nei Bagni. Su questo affinamento di ljuo v. Arch. IV, 404-5; al Morosi pareva invece che il suo figghiulu leccese fosse da -ōlus. Letterari sono qui figliole sing. masch., e figliolo.

Innanzi a vocale: puoy, voy e vuy (che forse è $v u(o) y)$; poe post, boe.

9. AU. Pl. provere, Muore Mauri.3 Auro, tresauro.

1 La casun ne' Bagni, v. 27I, andrà letto le casun.

Anche nel Regimen. Cfr. Me yer-Lübke, Ital. Gr., p. 42.

3 Puoco si ha nel Memoriale di Diomede Carafa. 
Vocall atone.

ro. A prot. caduta: poteca, strologia (astrolaco), miralglia e ammirante, stucia, Marfe 'Amalfi', Strune 'Astroni'. Prefisso: arribaudo, avanto 'vanto' sost.

In e-: esolvo se absolvo o non piuttosto exsolvo: ma è nel senso del primo; essencione 'ascensione', effeczione, estuto; Engioya e Ingioya 'Angio'. - Mediano: feceva anal. su fece, prematica, redificare 'ratificare'. Fut. e condiz. dei vb. in $a$ : derrd, penserrd, ferrd, derria, parlerrimo, troverrite, anderrite e andirrite ma fatraij, farrame. $\dot{\mathrm{E}}$ curioso che altri testi contemporanei, come il Cod. Aragonese, mostrino invece la tendenza opposta, conservando $a$ : pregard, mancard, lassarremo, pensarrimo ecc. Nei Bagni trovo tornard, tornerrai. Il Regimen ha derragio. Il dialetto moderno sempre $a$.

In o seg. da labiale: Dommasco, liobarde 'alabarde'. In $u$ : spruviere 'spar-'.

Ir. Post. Intatto nel pl. dell' imperf., mentre invece il Cod. Arag. ha esempi come amavemo, i quali gia mostrano avvenuta la ritrazione dell' accento: cfr. mod. -ávemo, -áveve, sull' anal. della $3^{2}$ pl. -áveno.

Finale. Per -eze che continua -ities, mogliere, lettere al sing. si vegga il mio lavoro in Ztschr. f. rom. phil. XXIV, 504. -unqua conserva la sua finale. Sopre da super, non supra; e questa voce, insieme con senpre, trascina seco infre, che ricorre accanto a fra.1 Il solito insenbra e e una volta insema.

Noto ancora raccza 'arazzo'.

12. E prot. caduta: rede, vangelie, visscovo, Giccziaca, lemmosena, rimmito. $D_{e-}$ si alterna con di-: quest' ultimo di regola quando segua nella parola un $i$ o anche $u$; es. deveva, divite; dereto, denare e disideruse, dilietto, dilito. Ma l'assimil. a $u$ non avviene sempre: es. defettuso, mentre è rarissimo che venga a mancare con $i$ : devimo. La medesima oscillazione è fra sengniore singniure, presiento prisiente (pl.); vestute vistile, venute vinire ecc. Da un lato nepote, decesselte (ma dicedotto), dall' altra procissione, filice, milodie, cirtissimo, rimmito, gintile, rigina (lett. regina, pl. rigine), ligite, cridiette, pirdio, abinimiento, sintimiento. Per analogia si finisce col trovare $e-i$ alternati nel sing. e nel pl., anche se non c' è assimilazione: fenesstra finesstre.

13. Spesso è sostituito da $a$, quando sia prossimo a $r$ : arro, arrata (ma errore e orrore per er-), arvayo accanto a erbayo, Sarapia 'Serapide', marcennaro 'mercenario', parlate 'prelati',2 pravarricao, fantaria, viernardy. Si vede che in varie di codeste voci è intervenuta l' assimilazione.

1 Così nell' ant. genov. sover, sovre, come enter, entre inter (Arch., VIII, 349). $E$ forse anche il nostro infre può esser foggiato su di un *intre. 2 Prelatri, parlati nei Bagni. 
Inoltre daffino 'delfino', malanconia; assiequio *exsequium per exsequiae, avolio 'avorio' e il solito piatate.

Conservato in appertene 'appartiene'. Assogliere sarà *adsolvere, e non -ex.

In $0:$ a contatto con labiali Sansoverino, dommonio (con assim.), 1 romanire; remonorare si risente fors' anche di onorare. Assim. in indonocchiato, ma anche inde-. Scambio di prep. in progidicio, prommetto 'perm.' Talora passa in $u$, sempre a contatto di labiale: Fuderico, dummanio, subburco sepulcru con assim., e cosi suplutura accanto a sobbellire e sibbillito. Qui intervenne anche lo scambio analogico con sub-.

Caduta mediana: vorrd e volerd. Si mantiene in venerd, apereva, Cepperano' 'Ceprano'.

14. Post. Arestotale; con assim. camara e camera.

Nel iato si dilegua in voci letterarie come tologia, Lonardo; ma liale riale viato 'beato', lo spagnolesco criato, riammo, accanto a beato, leato, leammo 'leg.', galeaccze; carreagi e carriaggie 'carriaggi'. Da aer airo; gaitane pl. m. Gaietani.

Finale. Non v' ha esempio d'infiniti in $-r i,{ }^{3}$ o d' avverbi in -menti, come in altri testi più antichi. $\mathrm{La}$ des. $-e \mathrm{pl}$. di $3^{2}$ decl. non ha nulla da fare con -es, come provano i mutamenti della tonica dovuti invece a un originario $-i$.

Noto la persistenza costante della finale nei verbi accompagnati da enclitiche: esserele, audireme, ecc., e negli avverbi (finale mente ecc.) che continuano a venir scritti in due parole staccate. Invece di qualche, sempre quale che. -E dell' inf. coniugato (v. Ztschr. $f$. rom. Phil. XXIV, 501) può esser sostituito da 0 , attratto dal -no della desinenza: esserono; ma più spesso rimane: essereno, faceremo. Cinco per anal. di quatto 'quatro'. Ante, oge conservano intatta la finale.

Epitesi di $e$ in este est (in frase latina) come nel Contrasto di Cielo dal Camo.

15. I prot. Aferesi: taliane, vierno, e in taluni composti con in: nante, dulgencia, namora; mala 'npresa.

Generalmente in e: ma trovando $i$ non si può inferire che la voce sia letteraria, trattandosi, in fondo, di una $f$ che si prestava facilmente all' equivoco. Lebberta, screvesse, merelariano, amecicia, vettoria, paresino 'parig.', deceva, esscrettura; nobbeletd, morteretale 'mortalità'; cosetore, serveture.

Dis - diventa des-.

In a: magliara, ass. contro il solito mi-; cosi incrimmanavano, ripracare 'replicare' e ripricare, sacralegio, sarvagia. Per l' it. 'Inglesi' si ha col manten. di a-angrise, come nel nap. mod.

1 domonio ha il cod. L. 10,42 (Naz. di Firenze) della lettera nap. del Boccaccio; demone in altri mss.

- Cipparano nella Cron. di Partenope.

- Es. esseri nella Croni di Partenope. 
In $o$ : Solivestro, con epentesi di $i$, come in indullicenzia acc. a dulgencia: cfr. l' it. filinguello. Cade nel gallicismo devengiare e in verilate, spagnolismo.

16. Post. Di regola $e$; ma puó esser conservato come quand' è prot., dall' assimilazione, o da una tendenza letteraria; e su ciò non giova insistere. Basti qualche rapido esempio. Poseto, preposito; abbilo; mirabbele, notabbele; sollecetutene, desordene, vergene: -issimo -essema, pl. -isseme -esseme. -I u sembra letterario: prubico, prubbica, frabiche.

Caduta interna: arma anima. Inserito in sisima 'scisma', Goglierimo.

In $a$ con assim. astraco, che è di vari dialetti settentrionali e meridionali; in -o pesole pensilis e, da $y$, martore.

In iato: vattiare; ma feura, veolato con $e$ second.

Finale: sempre $e$, anche ne' monosillabi (se, le illi) e nei verbi. Persiste nell' art. ly e in tutty, quasi sempre.

17. O prot. Spesso conservato: non solo in voci come poteca, ma anche zolite, consiglio, morio. Più popol. u: Ruberto; ottumano, furtuna, furtonato, scusute 'scuciti'; umayo, murio, curtisie, presunia, gruriuso, cuseture 'cucitori'. Quasi sempre fenomeni di assimilazione, come si vede.

In $a$ : anore, ma di solito on-; accaiso occasione, accziso, accisione, cainato, canossendo (seguo l' uso di notar qui questi fatti che andrebbero in tutt' altra rubrica).

Dissimilazione: delore, Belongnia, epposito ${ }^{1}$ preposito 'prop.' è scambio di preposizione come precura. Per assim. remmieo 'romeo'. Notevoli ammeruso, valleruso, il primo foggiato nel secondo, che a sua volta ricorda lo spagn. valeroso, foggiato nel verbo.2

Cade iniziale in staio 'ostaggio'. Inserito in Urobbano. Su Firincza, firintino.

Av. voc.: duana.

18. Post. Permane in arboro. - Filosafe, astrolaco, leparo.

Finale. Si conserva in como; e ricordo puro 'pure'. Una volta viau per vao 'vado'.3

19. U prot. Non meno di $\breve{\mathfrak{u}}$, anche $\overline{\mathfrak{u}}$ tende a esser sostituito da $o$, quando non lo trattenga l' assimilazione. Es. crodele, essfurtonato, furtonato, customato, sodario. Cum- dà co-; sub-so-.

Indenacchiate 'inginocchiati' altre volte è indeno-, per cui è probabilmente un errore.

Dissim. in securczo, remmore, sogecare subjug.; Picczulo (o Carafa.

1 Trovo apposito per opp. nel proemio al Libro delli precepti di Diomede

2 Valeroso ha il Di Majo; ameruso Del Tuppo; la stampa della Cr. di Partenope volunterosse.

S Una volta lore per loro, con evidente errore. 
Pecculo) Puteoli, corricsato 'corrucciato', prosincione; assim. in progidicio e forse in coliniello * columnellu (de fenesstra).

20. Post. - ulus -olo (puopolo, pl. masscole ecc.). - Qualche volta la $3^{2} \mathrm{pl}$. del pf. ha $-e$ per il consueto -o: essere exierunt, andare 'andarono', insorare-lo in uxoraverunt.

Av. voc. vettoaglia e vittuaglia.

21. Dittonghi. AU. omento 'aum.'; aucielle, audire-oderrite, laudaro, tresauriere.

In a: ascoterrit, arefece, Agusto, Agustino, arecchie.

EU: Eogenio, Eoropa, Oropa 'Eur.'.

\section{Consonanti.}

22. J iniz. generalmente intatto: $j a$, junto, judica, jodece, ecc.; gettare e jettaose. Med. maiure, peio; letterari saranno pegio, magesstade.

rj nelle voci popolari espunge il $\mathrm{j}$ : -arius -aro. A parte cuoiro.

vj: legiu; bj dà ǵ, anche dov' è scritto semplicemente $i$ : agio, sogecare, deio, aio, aloiano 'alloggiano'. Cons. + bj: scangio.

sj: presone; camisa, marvaso. Perusia accanto al pop. Perosa. Una volta vassare basiare, che di solito ha $-s-$. Da occasione acchaisone e accaiso. ${ }^{1}$ Nessun $\zeta$ da $s j$, come in più antichi testi. ${ }^{2}$

nj: ngn; sengniore ecc. Sonnalo è da *somnatu, cfr. Arch. IV, I6I. Accanto a lingnaio, si ha lilgliayo, con assim. progressiva; e così Alalglio ed Alangnio 'Anagni'. Dumminiava può ricordate lo sp. domeñar.

cj : ccz, suono di cui s' avrà a riparlare. Facczio, faccze facies; scauczo, cauczolaro, lancze; czod.

dj:g. Ogie (e oye), inguagiare (letter. inguadiare), assegiata ecc. Mangiare e mancziano. Mediu miecczo.

gj: letter. naufracio.

tj. Oltre le normali riduzioni italiane, noto i casi di $s$ per $c z$ : forsa, sensa (e sencza). ${ }^{3}$ Questa grafia non è infrequente nelle letteratura del tempo, e non può aver altro valore che grafico. Abbondano naturalmente forme semidotte come sacia, insacichliele, amecicia ecc.

Ptj: caccza, caccziata (caccziao e cassao); accuonczano e acconciate, accongiare.

pj: sacczo.

23. Merita un cenno a parte la determinazione del valore da attribuire al segno $c c z$ come continuatore d' alcune combinazioni

1 Regimen: accasone; Del Tuppo: accagione.

2 Cfr. Arch. II, 376n. e IV, 160 n. Nei Bagni, rascione.

3 De Jennaro, canz. I: alsarsi, sensa; Galeota: sensa, forsa, speransa, mensogna, cansone ecc. 
di $j$. Generalmente nei codici piu antichi si ha $\varepsilon$, del quale avverti il Mussafia che quando provenga da tj suona indubbiamente $t s$, secondo viene a confermare la pronunzia moderna. Invece, secondo questa, $\mathrm{cj}$ si risolve quasi sempre in $c^{\prime}$ : così che avendo presente il moderno $f a c^{\prime} c^{\prime} a$, p. es., non è chiaro come si debba leggere faccze, se con sibilante o palatale. E peggio sta la cosa per pj (mod. $\left.\operatorname{sac}^{\prime} c^{\prime} 0\right)$. Da consimili dubbi il Mussafia fu indotto ad attribuire al segno $\&(c c z)$ un doppio valore; e per il prodotto di pj ritenne trattarsi di $c$ '.

Ora, la grafia napol. del 400 conferma quel doppio valore di \& ccz. Osservo, anzi tutto, che questo segno è usato con tanta libertà da indicare suoni davvero disparati, se lo troviamo in mancziano' 'mangiano' che non sarà stato certo nè mantsiano nè manc'ano. ${ }^{2}$ Ma prescindendo da questi casi anormali, è frequentissima la doppia grafia acconciáte e accuónczano, Marcia e Marczia ecc.; -nce si alterna con -ncze: áve-nce, e-ncze. ${ }^{3} \mathrm{E}$ anche là dove non si abbiano codesti paralleli, basta il confronto con la pronunzia moderna a dirci che, p. es., cacczia, facczio, succzieso ecc. non potevano suonar molto diversamente dal nostro caccia, faccio, ecc. Ma v' è di più. Trovo ccz per la semplice palatale sorda latina: piaccze (e piacere), accziso (e accisionc), acczetto (e accetto), succzede (e succede).4

Chi volesse indicar la causa di tale confusione potrebbe essere tentato di scorgervi sotto una sfumatura fonetica; ammettendo nel napoletano antico un' influenza diffusa di quel cecear spagnuolo, così lungamente durato per le vie della città. Infatti anche oggi, chi ben guardi, codesta sfumatura interdentale o sibilante non è estranea al dialetto napoletano. E mi par difficile che uno scrittore come il Del Tuppo, infarcito di spagnolismi, quando scriveva caza, fazendo, faza faciat, faze facies non cercasse di rendere consapevolmente un suono affine a quello delle consimili voci spagnuole che gli servivan forse di modello. Spagnolismi crudi non sono, perchè troviamo qui $l^{\circ} z$ in tante altre voci dialettali; ma non troppo lontano dal suono spagnuolo doveva essere il suono napoletano. Contro questa ipotesi sta tuttavia il fatto che gid nel sec. XIV $c c z$ poteva valere $t s$ e $c^{\prime}$; quest' ultimo valore ebbe poi sempre a preferenza come continuatore di pj e cj.

Un' ultima prova del valore palatale ci viene del De Rosa

1 Nei Bagni è maniare. ital. IV

2 Cfr. manzare nel gliommero pubbl. dal Torraca, Giorn. stor. d. lett.

3 Del Tuppo: se 'ci', e nei Bugni si ha invece ge.

4 Cosi nel cod. della Cronaca di Partenope: Forcella e Forczella. Aggiungo qualche altro esempio che toglie ogni dubbio sul valore anche palatale di $c z, c c z$ in questi testi. Nel Sirventese del Maestro di tutte l'Arti (Ztschr. f. rom. Phil. V, 27) trovo in rima: sacczo (v. 181): lacczo : desfaczo : caccio : viaczo. E quel saczo diventa saccio al v. 103; così faczo (v. 60 riappare come faccio (v. 109.)

Nel cod. Riccard. 2752 una poesia spagnuola scritta da un napoletano offre feczios per fechos. 
medesimo. A un certo punto egli avverte: „Lo re aveva una figlia chiamata Sancsa, et nuy la chiammamo Cziancsia; dove il primo nome vuol essere evidentemente lo spagnuolo Sancha.

24. L. Da notare rapille 'lapilli'; otiritate 'util.', morteretate. Stuoro e stuolo.

Bossoda e bossola.

Se la grafia di tollere è dotta, più giova ricordare male tolleto: cfr. Paradiso V, 33.

I è rammollito in saglire, saglie.

Seguito da dentale, palatina o sibilante passa regol. in $u$ come nel nap. mod.: cauce, auto, fauccze, gaudo Wald, defauto 'diffalta'.

Questo $u$ non dilegua, anche se lo preceda altra voc. che $a$ : voucze, ${ }^{1}$ (accanto al cond. vócera e a volce). Viceversa dilegua sebbene preceduto da $a$ in alaro, accanto ad altaro. Inoltre suodo solidu e assodato 'assoldato'; utimo, utimatamente, la lutima con agglut. dell' articolo (e anche ultimo); mute multae; doce, docize 'dolcezze'.

In $r:$ cortellata, cortellessa ecc.: forse perchè in formola atona?

Innanzi a gutt. o lab. si ha $r$ : burpe vulpes, cuorpo colaphu, corpa, arcune 'alcuni', Burgarie.

Assim. a cons. seg. in daffino.

L' $\mathrm{R}$ vorrd vorria acc. a voleria.

25. PL: forme letterarie come p. es. piacere; ${ }^{2}$ ma chioppeto (part. forte di 'piovere'), schianato, acchiunpimiento - cumpl., ${ }^{3}$ con metatesi; chiummo. $\mathrm{Da}$ templu tienpo; da plenu pino. Esspenno, essprennam'nte 'splendid'. Semidotti essenpro, ripricare, prubico-plubico publ.; replubica.

CL dà chiammare ecc.; semidotto cremmencia, crerica, concruse.

GL gliandola, ${ }^{3}$ dov' dे evidentemente l; angrise.

Altri esempi di metatesi oltre quelli riferiti: carvacchao 'cavalcó', suplutura.

26. R. Dopo cons. dileguato in scontava 'scontr.', esspubbeduto 'sprovveduto'. Finale, scompare in quatto, soro. Fra vocali è, come si vedra dalla morfologia, costantemente geminato nel futuro; invece la doppia appare scempia in guera, tera, Engrettera, corevano, contre la più frequente conservazione. Frequente la caduta nel gruppo TR: terresto, maisto, esscuate (esscuatre), valeste 'balestre', pl. patrennuoste; oltre dereto. Patrinu patino, come nella lettera del Boccaccio; ma patre, pátremo.

Oltre il solito reqqueddere, va notato il $d$ in messtiede. Dissimilazione in gilifarco, odorifele: viceversa assim. in flagele. Dileguo

1 Divenuto poi vore (p. es. nel Basile); oggi sostituito dal pf. debole.

- Chiacere è nella lettera nap. del Boccaccio, e parve "grosso errore" al Galiani; ma chiaci ha pure il Contrasto di Cielo.

${ }^{3}$ Cfr. il romagnolo ciumpi, il sicil. chiumpiri. V. Arch. XIII, 362.

- Cfr. ibid., pag. 437. 
per dissim. in remmico 'romeo', pruopia (ma propria), arbitio (arrbilrio, arbritio).

Metatesi: stremminio, prommette 'perm.', Breongnia 'Borgogna', patrennuoste 'paternostri'; frabbica, preta; essturmiento ecc. Ricordo anche Coracie 'Croazia'.

Parlate 'prelati' sarà per infl. di 'parlare'. Notevole metatesi, se spontanea, inborcato 'broccato'.

Propagginazione di r: viernardy, bralestrata. - Calistro, 'Callisto', cilestre si risentono del suff. - $t r-$.

Fulto 'furto' è voce semidotta.

RS si assimila in susso (e suso), duosso, esscopesse 'scoperse'; muocczo morsu.

27. v. Salvo pochissime eccezioni, fra cui qualche $v$ iniz. geminato in $b b$, di cui avremo a riparlare, è raro il caso dell' alterazione iniziale, anche se precede una proclitica: come in le lurpe 'volpi'. Un pajo di volte trovo la prostesi: fo vuomo, in rira. 1 Epentesi di iato: paraviso, e una volta sola dove per il solito doe duae.

Frequente caduta interv.: saissimo 'sav.', pf. troao ecc.; vidanda 'vivanda'. Noto ancora Serie 'Serbia'.

Nella $I^{2}$ pers. del pf. deb. si ha il più sovente dileguo: alloay, tagliaime ecc., ma può anche mantenersi: pigliave, nutricave. Il mantenimento sembra esser di regola ne' verbi con $-i$ : vesstive, cusize; e, sull' anal. di questi, cadive, pussidive. - Dieve dẽdi ha subito influssi analogici (si veggano le note morfologiche).

28. W iniz. \& regolarmente $g$ o gu: gaudo wald, ganche 'guance', guera, inguadiare. Interno Suavia; treva potrebbe essere un gallicismo.2

29. F è geminato in maniffica, diffiette.

30. S. Lasciando il notissimo Cicilia, rimane intatto, fuor che in alcuni gruppi consonantici.

Ns: Sanczone, Alfonczo; Alfuncine, pencerrimo, pinciere. Sebbene il segno $c$ manchi di cediglia, vale indubbiamente $z$ (ds). Notevole cino 'seno'. Essendo preceduta dalla prep. in, l' iniziale è come se facesse parte del gruppo ns. - A parte vense.

LS, RS: faucza, pf. voucze (volce, volcero, cond. zolcera), touccze; fuorcze, muorczo, murczille, securczo, Corcica. Ma anche fuorse, persune, ${ }^{3}$ insenbra ecc.

sü: posse posuit.

Finale: cray, ey est, poe, po post.

ss è sovente scempiato dopo l' accento: miso, succieso, con-

1 Cfr. il nap. mod. vavone avu; come a Campobasso *vave ecc.

2 Nel Codice aragonese treuga.

s Perzona nella lettera del Bocc., come nel nap. mod. 
cieso, commiso, imprommiso, pf. procese (ma prociesso, grassa ecc.). Non entrano in questa rubrica per diversa ragione nè poccso * potjo nè vascio e vassio *bassiu.

31. Sc è scritto si, ssi o anche s: cussino, crissute, rencressie, dessiese, lassive; resusitaro.

Iniz. siendere, siese, sisero, siencia, sisima 'scisma'. Come si vede, a questa grafia non sfuggono nemmeno le voci semidotte. 1

32. X. Interv. ss: essenpro, esserrato, lassao. Forse sonò $s^{v}$ in esse exit, esseno; appoggiato a cons. insorao inuxoravit, insio -incio exiit.

33. ST sempre conservato, cfr. Arch. IV, 68 per i pron. chisso, chessa da -ipse e non da -iste. In rististe 'resiste', se non è errore, è influsso dell' ultima sillaba. Metatesi stentine 'intestini'.

Osservo, infine, che davanti a conson. abbiamo quasi costantemente ss per s: usanza non frequente nelle carte napoletane del tempo, ma di cui si hanno esempi fin dal trecento. Qualche volta sarà semplice abitudine grafica, tanto che troviamo, p. es., apersseno ecc.; ma forse innanzi a $k, g$, e a qualche altra cons. ss può corrispondere a $\xi$, in conformità con la pronunzia moderna.

34. N. Dissimilazione: arma anima, magnialimo 'magnanimo'. Assim. alimalia animalia in frase latina. ${ }^{2}$

Insire, ensire exire ha l' epentesi solo in forme arizotoniche: esse, ésseno: come in ant. genov. ecc. Nce 'ci' sull' anal. di nde inde. Vende per 'vide' é errore.

Costante la caduta nel gruppo Ns: appiso, spase ecc. Patiero 'paterno' sard errore; cfr. matrerno.

Assim. con l' iniz. seg. in bel-lo 'ben lo', bel-lavata 'ben l.', com-madamma ecc. Dubbio è nollo: essendo no assai più comunc di non, è probabile che sia qui no-llo, come ve-llo, me-llo ecc. $\mathrm{Ma}$ per questo, come per la geminazione di in-, v. n. 46.

Caduta: so sunt; renuccsao renuntiavit. $N e$ paragogico è raro: stane, tene, mene.4

35. Ar. Av. lab. costantemente $n$.

Assimil. notevole in cappare accanto a canpasse.

Normale la geminaz. interv. femmena (femena), Romma (Roma), comme (como), ammore, ammata, chiammare, lemmosene; fracetumme 'fracidume'. Per la $I^{2}$ ps. dell' ind. pr. v. la morfologia che seguirà. Combenda 'commenda' sarà per infl. di prebenda?

1 Del Tuppo: Sipione, septro, risignolo acc. a riscingiolo.

- Armalia nella Mascalcia del Rusio è voce popolare passata al sing. fem. Cfr. il portogh. armalha.

- Così generalmente nelle scritture volgari del sec. XV: ma nella Cron. di Part. leggo essuta presso insuta. In Del Tuppo il noto angonia per 'agonia'.

4 Tune nella lettera del Boccaccio. 
Sum ha perduta la finale: so. condana.

MN: onne, duonno ecc.; anche onuno, onipotente, e poston.

Noto da ultimo la geminazione anche in madamma, dammecella.

36. c. Al normale mantenimento iniziale fanno eccezione gattive captivi (anche in Del Tuppo) gossatao 'costo'. Qua e là si continua l' antica scrittura $c h$ per $k$, spec. innanzi ad $a$. Divien palatale in ciabrello, con alterazione non indigena. ${ }^{1}$

Interno, quasi sempre inalterato: luoco, poteca ecc. Casi di dileguo, passando per la sonora: preo, preano, preao (pregao), pae 'paghi', $2^{\mathbf{a}}$ ps. cong. pres.; paerrissevo 'paghereste', alloay.

CR che ci aspetteremmo sempre intatto, scade sovente a $\mathrm{gr}$ : groce, grisstiane ${ }^{2}$ voci letterarie saranno grocta, grasse, sagra ecc.

Cr è sempre, anche graficamente, $t t$; salvo che in dilito. Dal suff. -ATICU sarvagia, lingnagio, ecc. e gli equivalenti lingnaio, umayo, stayo obstaticu ecc. Trovo aio de fatica nel senso (rivelatoci dall' autore medesimo) di tienpo de fatica: dunque un gallicismo per age.

Devengiare 'vendicare' (prov. venjar) è entrato fin delle origini nella nostra lingua letteraria.

37. QU. Noto cinco, cercua quer., cuoce 'cuochi'. L' elemento labiale permane in qua, che nel nap. mod. è $c c d$; dilegna pur costantemente nei pronomi: chisto, chello ecc.

38. c'. Abbiamo avvertito che $c z$ può anche servire a rappresentar la palatale sorda. Si ponga mente alla triplice grafia eccelsa, esselczo, ecchelczo. Quest' ultima maniera spagnuola ricorre in ganche 'guance', e la ritrovo in una lettera della regina moglie di Ladislao: ${ }^{3}$ bichirilli, vichini (del resto, fin nel Regimen già si trova picchuni). Quanto a esselczo, mi ricorda mersede del de Jennaro (canz. I), merse ibid. son. XVI, come Barselona in Del Tuppo. Lo spagnuolo deve esserci entrato.

Cetto 'zitto' sarà in rapporto con lo sp. chito, meglio che con le analoghe forme settentrionali italiane. Per citella rimane il dubbio che ebbe il Mussafia sulla pronunzia del suo citelli; la lettera del Boccaccio ha zittielli, il moderno vap. sito. Bisogna dunque pensare che sia in realtà $\varsigma$, come Sviceri in Del Tuppo.

Agise 'uccise' dev' essere errore per aczise. Dammecella e paresino 'parigino' trattano diversamente $s$ francese. Popolare è aucielle *avicelli.

39. G iniz. av. $a, o, u$ si fa facilmente sorda: covernatore, covernacione, calee (galea). Interv. o segue la medesima vicenda o

1 Si conferma cosi l' opinione del Mussafia che nel çabrelli del Regimen vide "ein sekundäres Laut, wohl $\varsigma$, so dafs die doppelte Funktion des Zeichens $\xi$ immer wahrscheinlicher wird " (n. 64).

- Nap. mod. croce, cristiane.

3 Pubbl. dal Capasso, Ancora $i$ Diurnali di Matteo Spinello da Giovenaseo, Firenze, 1896, p. 25. 
cade: streca, casticato; feura, briantino, duana, briata, Raona 'Aragona', leammo (legato), Breongnia 'Borgogna'; vae 'vaghe'.

In sillaba fin. di proparossitono astrolaco.

40. GN dà il solito meridionale cainato; ma reino accanto a renno non mi par sicuro: potrebb' essere uno spagnolismo. Generalmente la grafia $n n$ : renno, dinno, sinne, o $n$ : rend, renao, assinare. Naturalmente si sentiva qui la grafia spagnuola. Infine, si ha sovente $n g n i$ o $n g n$ : singnio ecc.

$\mathrm{G}+$ cons. cade in Matalena, prena, treva, frito (se non $\mathrm{e}$ errore per friddo)

4I. G'. Non m' è chiaro jiste 'giostre', cui non basta a spiegare un influsso di 'geste'. Noto il gallicismo joetle.

Interv.: regina, legie ecc.; reggina; saettia, liey 'tu leggi', accanto a liege, fuire, corree, faito, maistro. La pronunzia di paio è indicata dal corrispondente pagio; in santo Giorio (mod. Jorio) ha parte la dissimilazione. $D$ per $G$ in indenocchiato.

Non andrebbe qui ricordato il pl. cioppe, che corrisponde pel significato all' ital. cioppa, sp. chupa (Diez, Et. W. I) mentre l' arabico giubbone, sp. jubon ecc. è rappesentato in Del Tuppo da juppone. 1

42. T è sempre mantenuto. Tando su quando, per la comune significazione temporale (mod. tanno 'allora'). Sadesfare 'sodisfare', redifico 'ratifico' non sono che storpiature ingenue di voci letterarie. Su frado v. il n. 3. Decedotto 'diciotto' sarà da decem-et-octo (Arch. IV, 147 n.) o non ha il $d$ in epentesi di iato come vidanda? - Normali sono spata, strata, curreluro ecc.; criado passando dalla Spagna s'è visto ristorare la sorda in criato; spagnolismo è desscreo.

- tate è sol raramente ridotto a -td (cild ecc.): potesstale, metale, citate (citatine) ecc. Verdate è forse spagnolismo: ma lo troviamo già nel Regimen, e questo fenomeno del $\mathrm{rt}$ in $r d$ è comune, con altri dialetti, al napoletano, sebbene il testo qui non ne dia altri esempi; ${ }^{2}$ chè viceversa troviamo bombarta. - nt non si trova ridotto nd: ma per tal trafila sard passato quann' quantu (quann' at-le 'quanto a te'). - Ante, nante continuano direttamente ante; ma altre scritture del tempo presentano forme diverse. ${ }^{3}$

$\mathrm{Da}$ tu: potte pf. - Auttro non è che un capriccio grafico; $t$ inserito in Istrael.

43. D iniziale si mantiene, alterato, in tarcenale 'arsenale', voce comune in tutto il ' 400 , che ricorda il sic. tirzand, lo sp. alarazanal e il nap. mod. tárcera (cfr. ital. darsena).

1 Non ho trovato nel De Rosa inregno, come nel Cod. Aragonese e nelle rime del Galeota.

${ }^{2} \mathrm{Ma}$ veri spagnolismi sono verdatero nel De Majo, c. XI, e verdatiero nel cod. XIII AA 39 della Cron. di Parten. (Nazionale di Napoli).

3 Innanci, innance, nance. 
Interv. cade facilmente: Corrao e Corradino; creo, deo dedit, Surapia 'Serapide', para(v)iso; vao, vaa, vae, e con $g$ epentetico vaga. ${ }^{1}$

Inoltre veo, veano. Obberiamo per obbede-. Passaggio alla sorda in agratevele 'gradevole'.

ND raramente si assimila: donne deunde; desideranno, contro i soliti gerundi con $n d$. Un addd, fra moltissimi andd, è errore. Da splendidu, essprennu e esspennamente.

D secondario gemin. in requeddere.

44. P iniz. conservato in poteca; conservaz. int. in voce letteraria arcepisscopo, e inoltre recepio, receputo. ${ }^{2} \mathrm{Ma}$ popolare è sape. - In $-b-:$ sebbellire, abbrile, subburco sepulcru, ciabrello. - In -v-: pariglione.

La doppia è scempiata in popa 'poppa', forse non indigeno. PT: grocta, grutle; semmana ricorda lo sp. semana; è anche mod. Suberbia, se non è spagnolismo. è dovuto ad assimilazione.

45. B iniz., il più delle volte $v$ : vandere, valeste, varva, vando, vasarosse, vialo, vevere (bevere), vossco (bossco). Ma bossoda 'bussola', boccole. Interno fra vocali sempre $v$ : sivo 'sego', trivolo. Erbayo è letter. accanto a arvayo.

Nell' imperf. è rara la caduta del $v=\mathrm{b}$ (v. la morfologia).

Frequentissima la geminazione: con -abilis ecc. si ha insaciebbele, notabbele, piacebbele, mirabbele; e, più rari, piacevele, rencressivele. A parte possebele. Inoltre: Robberto, jebbeleo 'giub.', libberao, robba ecc.; libberea 'livrea'.

$\mathrm{MB}$ in mm: gamme, trommelta, chiummo - piummo (chiumbo) plumbu. Viceversa trobbetta, abbassiature 'ambasciatori'.

BU: appe habuit, veppe *bibuit.

Un' ultima considerazione merita Ottrufo, metat. per Oltufro octobre. Non è oggi forma viva, ch' io sappia, nè saprei darle riscontri oltre le note forme col suffisso osco-sabellico -fru. Cfr. Arch. X, 1-17.

46. Geminazione. I più comuni casi di raddoppiamento sono stati accennati; in quanto a rr nel futurn, si vedra la Morfologia. Noto che ne' composti con ad- si ha raramente l' assimilazione (abbracczaro ecc.), e più spesso il $d$ scompare: acordado, afatucchiarie 'fattucchierie', aconpangniärele, atendeva ecc.

In generale il cod. è ricco di esempi di raddoppiamento del' iniziale dopo taluni monosillabi:

et: et ppiglia, et ppo, et ddarele. Naturalmente il $t$ si scrive per pura abitudine grafica, sebbene in realta si trovi assimilato nell' iniziale: ep-piglia ecc.

da tte.

1 Quando dice vaga l'uno per l'autro, si potrebbe pensare a un 'valga'; ma altra volta si legge vaga proprio nel senso di 'vada'.

- Recipir in Del Tuppo è uno spagnolismo. 
per ffine; che gia era, come oggi, pe ffine, o meglio pef-fine. in: probabilmente poteva non avvertirsi la pronunzia di $n$ : altrimenti non si spiegherebbero in pprima, in gguardia, in Sscavonia 'Schiavonia'.

con thutta (mod. co'). Inoltre: co Mmaria, co mmadamma.

che raddoppia non solo da quid, ma anche da qui, indistintamente: che mme, che sso, che llegile ecc.

Fin qui si è trattato di assimilazione. Invece è raddoppiata veramente l' iniziale nei casi seguenti:

lo ffiglio, lo pprisiento, la bbella, le pporte, le ttere.

de Llorito, de mme, de Nnavara, de Ppetrucczo.

Non ho contato esempi come fra lle, infre lle, potendo essere continuazione della doppia di ille ecc.

47. Passando ora alle voci polisillabe, non trovo di regola geminazione dell' iniziale prodotta da precedente parola ossitona. Invece oltre alcuni radoppiamenti prodotti da como ed uno (como fforo, una vverga, uno ccane), trovo una piccola famiglia di esempi isolati che sfuggono a qualsiasi determinazione: zidite ccomo, carosa dde te, picczolo ttienpo, in Gaeta qquatto, ly figlie sse rebbellaro, to fece sstrassinare, lassa ffare, illustrissimo dduca, sengnore ttuo, tutty ppresune, fece ppartire.

Qualche volta si possono trovare le forme oblique del pronome con raddoppiamento della conson., anche se appoggiate a voci parossitone o proparossitone: sapendo llo, facczeva llo, preao lle, prese lle, portano tte. Di queste forme, le quali ricorrono anche in Del Tuppo, il Mussafia trovò alcuni esempi nel cod. A del Regimen. Ma da ille non è a parlar di geminazione; quanto a tle, si può convenir col Mussafia che sia difficile attribuirgli un valore fonetico.

(Fortsetzung folgt.)

PaOlo Savj-Lopriz. 